\title{
El estudio de los saberes agrícolas como alternativa para el desarrollo de las comunidades cafetaleras
}

\author{
Anabel Martínez-López ${ }^{1 \S}$ \\ Artemio Cruz-León ${ }^{1}$ \\ Dora Ma. Sangerman-Jarquín ${ }^{2}$ \\ Salvador Díaz Cárdenas ${ }^{2}$ \\ Joel Cervantes Herrera ${ }^{3}$ \\ Benito Ramírez-Valverde ${ }^{4}$
}

${ }^{1}$ Desarrollo Rural Regional-UACH. Carretera México-Texcoco km 38.5, Chapingo, Estado de México. CP. 56230. Tel. 595 9521500. (etnoagronomia1@ gmail.com). ${ }^{2}$ Campo Experimental Valle de México-INIFAP. Carretera Los Reyes-Texcoco km 13.5, Coatlinchán, Texcoco, Estado de México. CP. 56250. Tel. 55 38718700, ext. 85353. (sangerman.dora@ inifap.gob.mx). ${ }^{3}$ Centro Regional Universitario Oriente-UACH. Carretera Huatusco-Jalapa km 6, Huatusco, Veracruz. CP. 94100. Tel. 2737340764. (disalvar1@yahoo.com.mx). ${ }^{4}$ Centro Regional Universitario Centro Norte-UACH. Cruz del Sur núm. 100, Constelaciones, Zacatecas. CP. 98060. Tel. 492 9246147. (jcervantesherrera@yahoo.es). ${ }^{5}$ Colegio de Postgraduados-Campus Puebla. Carretera Federal México-Puebla km 125.5, Colonia Libertad, Puebla. CP. 72130. AP. 2-12. Tel. 222 2851442. (ramirez@ colpos.mx).

§Autora para correspondencia: any1902@ hotmail.com.

\section{Resumen}

Los saberes agrícolas son un bagaje de conocimientos ancestrales generados por los campesinos; a través, del tiempo para el uso óptimo de sus recursos naturales. Hoy en día, en muchas regiones de nuestro país, donde todavía se practica la agricultura tradicional, se puede observar la utilización de estos saberes. Ante la política pública que impone la modernización de la agricultura, mediante programas de asistencia técnica, impulso de paquetes tecnológicos e incentivos a los productores, los resultados evidencian que no se ha logrado el desarrollo esperado y los campesinos son cada día más pobres. El sector cafetalero se encuentra en esta situación, a pesar de producir un cultivo comercial. Las estrategias impulsadas se han enfocado al incremento de rendimientos, con fines de mejoramiento económicos; sin embargo, para los pequeños productores tradicionales, tiene otros significados. Con la intención de explicar esta situación, el objetivo fue analizar documentación pertinente a la búsqueda de alternativas para el desarrollo agrícola considerando las necesidades específicas, recursos y visión de los productores. En contraste con el pensamiento hegemónico de la ciencia occidental, se construyó un marco teórico para la construcción de propuestas tomando en cuenta las epistemologías del sur. Se parte de la premisa que es posible hacer alternativas exitosas para el campo; a través, de un diálogo de saberes entre los poseedores locales y los estudiosos de la ciencia moderna, para lograr un desarrollo agrícola, comunitario o bien llamado etnodesarrollo.

Palabras clave: conocimiento ancestral, diálogo de saberes, etnoagronomía, etnociencia, etnodesarrollo.

Recibido: julio de 2019

Aceptado: septiembre de 2019 


\section{Antecedentes}

La enfermedad de la roya del café (Hemileia vastatrix Berk \& Br.) es un problema estudiado desde su aparición en México se ha intentado controlar enfocando la inhibición del hongo como objetivo principal; a través, de la ciencias agronómicas, que centran su control en la sustitución de variedades actuales por nuevas que presentan resistencia y control por método químico, por lo se incrementan los costos de producción, razón por la cual no es solución para todos los cafetaleros del país, ya que la producción de café se distingue por estar presente en unidades descapitalizadas y con superficies pequeñas.

Se tiene conocimiento que se registró un primer ataque por roya en México en 1981 (Escamilla, 2016), sin embargo, en los primeros años los daños fueron imperceptibles en la producción. Hoy en día, Hemileia vastatrix está presente en todos los estados cafetaleros del país con severa afectación en las cuatro principales entidades productoras de México.

Los daños que causa la roya, en conjunto con la crisis económica que prevalece en el sector, han conducido a las cosechas más bajas en los últimos cincuenta años; en el ciclo 2012-2013 se obtuvieron 5.1 millones de sacos (MS), en 2013-2014 la producción fue de 3.1 MS, para el 201415 de 3.6 MS y, en el ciclo 2015-2016, de tan sólo 2.3 MS (Escamilla, 2016).

Otro indicador de los graves daños que provoca esta enfermedad del café se observa a nivel de la productividad por hectárea, en 1976 se registró el promedio más alto en la historia del café mexicano, con 16 quintales por ha. Posteriormente, siguió una caída sostenida en las últimas dos décadas, con niveles entre 7 a 8 quintales por ha, en el ciclo 2014-2015 el promedio fue de 7.5 y cae a 4 quintales por ha en la cosecha 2015-2016 (Escamilla, 2016).

Desde que el grano de café fue sujeto del libre mercado, los productores se enfrentan a una fluctuación incontrolable de los precios del aromático, de donde derivan la inaccesibilidad a los mercados, bajo índice tecnológico, escasa asesoría técnica, poca mano de obra, rendimientos bajos debido al ataque de plagas y enfermedades y por ende, bajos ingresos (Cardeña, 2017).

Los saberes y la agricultura tradicional juegan un papel importante en la construcción de nuevas propuestas de desarrollo. Los campesinos como productores de café desarrollan conocimiento a través del tiempo para adaptarse a los cambios, tanto ambientales, como económicos y sociales (Beristain, 2001).

Desde el punto de vista cultural, destaca el hecho de que alrededor de este cultivo existe una gran riqueza y diversidad de valores, creencias y conocimientos que es necesario reconocer y estudiar (Moguel y Toledo, 1996), en virtud a que son dichos conocimientos los que han permitido resolver los problemas que se presentan en la producción.

La etnoagronomía es una etnociencia que se encarga del estudio de estos saberes que los campesinos indígenas y mestizos ponen en práctica durante los procesos de aprovechamiento de los recursos naturales (Cruz et al., 2015b) y la continuidad en la construcción de esta disciplina está enfocada al desarrollo agrícola. 
En México el café se produce fundamentalmente por pequeños productores, generalmente de comunidades indígenas o mestizas tradicionales, lo anterior es resultado de la historia agraria y cultural del país, donde la sabiduría indígena se apropió literalmente de un cultivo exótico, para adoptarlo y adaptarlo a los sistemas agroforestales nativos (Moguel y Toledo, 1996).

Por ello, la producción de café y la población indígena se encuentran íntimamente ligados, en las estadísticas los indígenas como productores de café tienen mayor presencia en el ámbito nacional, de ahí que la atención a los cafeticultores no puede ser solo desde las políticas de desarrollo rural, se debe considerar que cualquier política dirigida al fomento productivo de café, debe estar asociada a una política indigenista (Robles, 2011).

Los campesinos a lo largo del tiempo han experimentado con el conocimiento empírico tradicional, obtenido a partir de su cosmovisión y la observación de los componentes de su medio, para lograr un equilibrio entre la producción y la conservación de los recursos naturales. En sus sistemas agrícolas tradicionales, particularmente el agroecosistema cafetalero, se caracteriza por tener una aplicación de conocimiento que se ha transmitido de generación en generación, los campesinos cafetaleros han creado el conocimiento suficiente para hacer frente a los problemas que se desarrollan dentro de sus cafetales (Cruz y Torres, 2015).

Por lo anterior, resulta importante preguntarnos ¿Cuáles son los factores ambientales y de manejo de las plantaciones que están favoreciendo el impacto de la roya del café en la región de Huatusco, Veracruz, y cuáles son los que lo atenúan? y ¿Qué innovaciones se pueden aplicar mediante una estrategia participativa, combinando las tecnologías tradicionales y los avances científicos, para disminuir los efectos de esta enfermedad?

Para responder estas preguntas es importante sentar las bases para una mejor comprensión del problema, por lo cual, este ensayo tiene el objetivo de construir un marco teórico que permita la fundamentación de los saberes de los campesinos, como ciencia endógena, pertinentes al manejo de la roya del café.

Se analiza la información de contexto de las políticas públicas relacionadas con café en México, la enfermedad de la roya en el país y los resultados de investigaciones realizadas por los expertos en el tema de saberes locales, también llamado ciencia endógena, su aplicación y como han resultado en proyectos de etnodesarrollo o desarrollo agrícola en el contexto de lo rural, enfocado a las regiones cafetaleras.

\section{La decolonización y la epistemología del sur}

A mediados del siglo XX algunos estudiosos de ciencias humanas Lévi-Strauss (1972) pusieron en debate a científicos occidentales sobre la existencia de saberes indígenas sobre la naturaleza y sus sociedades. Ante tal evidencia, algunos autores después de Lévi-Strauss han señalado la necesidad de legitimar, sistematizar, escribir, formalizar o convalidar los saberes tradicionales, asumiendo que se requieren para ello instrumentos de la ciencia occidental (Pérez y Argueta, 2011).

Con el paso de los años este concepto fue evolucionando, en México se le ha denominado como sabiduría popular, saber local, folklore, ciencia indígena, conocimiento campesino (Pérez y Argueta, 2011) y con ello, la lucha para lograr su valorización al rescate de los recursos naturales, 
que la actual sociedad con su estilo de vida dominantemente occidental pronto hará entrar en colapso ecológico y civilizatorio, lo cual tendrá consecuencias para el medio ambiente y para la ciudadanía mundial más pobre y vulnerable (Collado, 2016).

Dado que cada forma del conocimiento simultáneamente tiene una dimensión local, regional y global, Delgado y Rist (2016) señalan que el concepto de saberes locales no tiene sentido ni fundamento epistemológico, además de que tiene una carga excluyente y discriminativa. Por lo que, en lo sucesivo, se referirá al concepto con sus otras denominaciones.

De Sousa (2011) aborda esta idea desde la epistemología del Sur, plantea que esta corriente son el reclamo de nuevos procesos de producción, de valorización de conocimientos válidos, científicos y no científicos y de nuevas relaciones entre diferentes tipos de conocimiento, a partir de las prácticas de las clases y grupos sociales que han sufrido, de manera sistemática, destrucción, opresión y discriminación causadas por el capitalismo, el colonialismo y todas las naturalizaciones de la desigualdad en las que se han desdoblado.

El sur es usado aquí, como metáfora del sufrimiento humano causado por el colonialismo y el capitalismo. Es un sur que también existe en el norte global geográfico, el llamado tercer mundo interior de los países hegemónicos. Al mismo tiempo, el sur global geográfico contiene en sí mismo, las prácticas locales de complicidad con aquellos. Tales prácticas constituyen el sur imperial. El sur de la epistemología del sur es el sur anti imperial (De Sousa, 2009).

Esta visión epistemológica conocida como epistemología del sur se caracteriza por albergar un diálogo horizontal con los conocimientos de los subalternos colonizados en una ecología de saberes. Entiéndase por ecología de saberes un abordaje epistemológico que abarca los saberes no científicos subyacentes en las culturas ancestrales, la sabiduría indígena, las artes y otras formas de organización del conocimiento que incluyan las inter-retro-acciones del género humano consigo mismo, con el otro, con la naturaleza y con la totalidad cósmica que sustenta lo sagrado (Collado, 2016).

A partir de esta base teórica resulta importante potencializar el conocimiento ancestral combinado con la razón científica en la perspectiva del desarrollo de las comunidades rurales en donde están mayormente presentes estos aspectos espirituales, religiosos, emocionales, retóricos y artísticos. Tomando en cuenta que el concepto de desarrollo no es asimilable a la idea occidental de progreso continuo hacia el bienestar, sino más bien a un modo de vivir el presente en armonía con la naturaleza, como lo plantea la noción del Buen Vivir (Vanhulst y Beling, 2013).

Autores como Collado (2016), refiere a que el diálogo con la sabiduría indígena y aborigen nos permitirá desarrollar nuevos horizontes epistemológicos más resilientes. Al respecto, De Sousa (2011) dice que, para las epistemologías del sur, el individuo autónomo es un producto de comunidades autónomas, y las comunidades autónomas no afirman su autonomía negando la naturaleza, sino todo lo contrario, asumiendo ser parte de ella, de esa madre tierra y en armonía con ella, como forma de sostenibilidad de la vida. A contrapunto, la supresión forzosa de conocimientos indígenas y ancestrales llevada a cabo por la colonización europea, que todavía continua hoy en día con nuevas formas neoliberales, es una forma de 'epistemicidio' que empobrece al ser humano en su totalidad (Collado, 2016). 
Los saberes tradicionales, entendidos como los conocimientos que han sido generados, aplicados y utilizados por comunidades y pueblos tradicionales, como los grupos indígenas de América Latina, constituyen una parte medular de las culturas de dichos pueblos y tienen un enorme potencial para la comprensión y resolución de diferentes problemas sociales y ambientales.

Esto, no es característico solo de nuestro continente, en África, Asia y en la misma Europa, existe un enorme reservorio de conocimientos tradicionales, por lo cual todos los países, incluso los más avanzados como los europeos harían bien en revisar sus políticas con respecto a los conocimientos tradicionales, para impulsar su preservación y aprovechamiento social, por ejemplo, los relacionados con la agricultura (Olivé, 2009).

\section{La existencia de conocimientos y saberes de los indígenas y campesinos}

En México, las zonas cafetaleras coinciden con los territorios de varios grupos indígenas y donde se practica una agricultura campesina a pequeña escala, González (2006) describió un caso ejemplar sobre la producción de café a pequeña escala en las tierras altas de Chiapas, la cual, se inició en la década de 1960 como resultado del abandono del cultivo de caña de azúcar.

Estos campesinos indígenas cultivan el café conservando la vegetación natural, es decir, en interacción con el bosque caducifolio, constituida por pinos, robles (Quercus Skinnerii, Q. acatenangensis, $Q$. candicans, $Q$. oocarpa, $Q$. corrugata) y goma dulce (Liquidambar). Como parte de su conocimiento ancestral, conservaban simultáneamente el maíz como policultivo, de donde obtenían frijol, calabaza, chile y habas, además de otras plantas comestibles y medicinales (González, 2006).

En México, más de $98 \%$ de la superficie ocupada por el café se cultiva bajo árboles de sombra: sistema especializado, policultivo tradicional y comercial y sistema de montaña (Escamilla y Díaz, 2016). Lo cual significa, que también prevalece la cultura de diversificación de los cultivos con el fin de obtener diferentes productos de las parcelas, además del cultivo principal, y que ha sido una estrategia de supervivencia de las familias para abastecerse de alimentos a lo largo del año.

Sin embargo, el elemento que sustenta la marcada diferencia en sistemas de cultivo es la polarización en el tamaño de los predios por cafeticultor. Así, 92\% de los productores tienen hasta 5 ha en producción; mientras en el otro extremo, $0.1 \%$ de más de 50 ha, constituyen el reducido grupo de grandes finqueros. Cabe aclarar, que la relación no es directamente proporcional, en el sentido de que, a mayor tamaño de predios, más intensificación en las prácticas agrícolas en todos los casos. Existen plantaciones pequeñas, intensivas en el uso de la mano de obra, con resultados interesantes, la producción de café orgánico en México, por ejemplo (Escamilla y Díaz, 2016).

Esto demuestra que, aún en estos días, en el sector cafetalero predomina el cultivo del aromático en pequeña escala y las unidades de producción utilizan mayormente la mano de obra familiar, valiéndose de tecnologías que han sido parte de la vida cotidiana de estas familias cafetaleras, practicadas desde la introducción del cultivo en México.

De acuerdo al Censo Agrícola, Ganadero y Forestal (CAGF, 2007), en los estados cafetaleros de Chiapas y Veracruz, de un total de 780979 unidades de producción cafetaleras, $15 \%$ de ellas se ubicaron como mecanizadas, en tanto que $67 \%$ de ellas solo usan herramientas manuales (INEGI, 
2009). Se tiene un cultivo basado en la mano de obra, debido a que la tracción mecánica en las unidades de producción, explicable en términos de los requerimientos de manejo del cultivo, las condiciones abruptas en donde se realiza y que las unidades de producción son pequeñas que se encuentran en condiciones de pobreza.

Derivado de los problemas ocasionados por la enfermedad de la roya del café, las medidas implementadas por el gobierno mexicano apuntan hacia la renovación de cafetales con variedades resistentes a la roya, además del uso desmesurado de agroquímicos, principalmente fungicidas.

La respuesta de los productores ha sido positiva, ya que se promocionan como variedades, resistentes a la enfermedad, de altos rendimientos y con bajos requerimientos de sombra; situación que no ha valorado a los cafetales tradicionales como corredores biológicos y reservas de la biosfera y dado el cambio de variedades provocarían la desaparición de especies de flora y fauna que interactúan de manera natural dentro de los cafetales sombreados (Escamilla, 2016).

Las variedades resistentes a la roya que se cultivan en la mayor parte de los países productores parten del híbrido de Timor como material genético, el cual es una hibridación natural entre Coffea arabica y Coffea canephora. De acuerdo a Arrieta (2015), este híbrido ha sido utilizado en los principales programas de mejoramiento genético del café a nivel mundial.

Actualmente, estas nuevas variedades están perdiendo resistencia contra la roya, además de que generan granos de menor calidad en la bebida en comparación con las variedades tradicionales (Typica, Bourbon, Caturra) y son responsables de la dispersión de nuevas enfermedades en los cafetales que antes no se tenían.

Al respecto Pérez y Ruíz (2017) opinan que, al apostarle al cambio de variedad, es decir, la siembra de Catimores y especies provenientes de Costa Rica y Colombia, se generan también problemas sociales como: acaparamiento de las mejores tierras por empresas transnacionales cuando los productores ya no cuentan con ingresos para su supervivencia, migración, desintegración familiar, incremento de inseguridad, e índices delictivos y producción de cultivos ilícitos.

Este es el motivo para lograr nuevos proyectos de desarrollo rural con la participación de comunidades campesinas, atendiendo necesidades específicas de los productores por regiones, que resulten en una mejora de las condiciones de vida y bienestar de las familias cafetaleras.

Dado que los campesinos se apropiaron del cultivo de café hace más de doscientos años, esta actividad productiva, que incluye el cultivo y la organización del trabajo, lleva implícita una dimensión cultural. Ejea (2009) dice que aun cuando todos los productores de café comparten los mismos procesos generales; es decir, condiciones técnicas de la producción cafetalera, sujeción a una fragmentada cadena productiva y vaivenes del mercado mundial, existen diferencias en los contextos particulares y las formas de apropiárselo e incorporarlo a las dinámicas sociales y económicas del entorno inmediato.

Sobre todo, varía de región en región e incluso de comunidad en comunidad, por lo que, los proyectos direccionados a los pequeños productores de café tendrían mejor efectividad si tomarán en cuenta estas diferencias, principalmente del manejo tradicional y cosmovisiones en cuanto al vínculo del cultivo con los productores y sus intereses. 


\section{De las etnociencias a las ciencias endógenas}

Los saberes tradicionales, también llamados sabiduría popular o conocimiento campesino (Argueta, 1997), son todos aquellos conocimientos adquiridos de manera empírica; a través, de miles de años por los pueblos antiguos para el aprovechamiento óptimo de los recursos disponibles en su medio, desde la domesticación de plantas y animales, invención de herramientas de trabajo, hasta la selección de variedades, lo cual se logró con la la observación y experimentación con base a su utilidad alimenticia, medicinal o de vestimenta.

El estudio de los saberes tradicionales está a cargo de la disciplina nombrada etnociencia, la cual, tiene el objetivo de estudiar ideas, procesos y formas de relación, bajo las dimensiones de tiempo y espacio entre los pueblos o poblaciones humanas y las especies y ecosistemas (Pérez y Argueta, 2011). La ciencia durante siglos ignoró la existencia, características y potencialidades de la sabiduría de los pueblos indígenas en América.

Latina y el Caribe y las etnociencias han formulado una propuesta teórico-metodológica para estudiar y analizar los saberes. Por lo anterior, se parte de que existe un conocimiento profundo sobre los procesos productivos, que se ostenta, la sabiduría campesina. En el caso de los productores de café, se expresa en el reconocimiento de las particularidades del clima y sus efectos sobre las plantaciones en sus diferentes etapas.

Las técnicas desarrolladas en años por los campesinos indígenas, forman parte de lo que Hernández $\mathrm{X}$. nombró como tecnología agrícola tradicional y que definió como aquellos elementos culturales emanados del conocimiento empírico acumulado por las etnias rurales durante miles de años, en sus intentos de utilizar los recursos naturales renovables por medio de las explotaciones agrícolas, pecuarias, forestales y faunísticas para obtener los satisfactores antropocéntricos para su subsistencia y desarrollo social y económico (Hernández et al., 1976).

Existe evidencia acerca de la implementación de estas tecnologías en el medio rural; en el caso del cultivo de café, en un estudio realizado en la sierra de Zongolica, Veracruz, se encontró que en los cafetales los instrumentos empleados son simples y manuales, algunos son adaptaciones de los introducidos por los españoles y otros son mesoamericanos modificados, como el cavador. Los suelos para cafetales se preparan mediante la roza-tumba-quema o sus variaciones, es decir, se encontraron parcelas en las que solo se rozó-tumbó, se sembró maíz y entre las hileras de este cereal se trasplantaron plántulas de café (Dzib, 1994).

En muchas regiones cafetaleras del país estas prácticas siguen vigentes, también la utilización de herramientas manuales como mencionan los autores tal es el caso del machete o 'moruna', azadón, pala, ahoyador y otros instrumentos adaptados y modificados por los productores para su utilidad, además nombrados localmente. Estas tecnologías forman el campo de estudio de la etnoagronomía, entendida esta como la etnociencia que basa sus estudios en las visiones y aspiraciones de los productores con persistencia en saberes tradicionales relacionados con el conocimiento de los recursos naturales, la diversidad de cultivos, especies silvestres aprovechadas, tecnologías de manejo, herramientas tradicionales, rituales, calendarios agrícolas y formas de transmisión del conocimiento (Cruz et al., 2015a). 
Es necesario pensar en un desarrollo basado en los pueblos y la gente que los integra, distinto al desarrollo occidental, en donde los pueblos indígenas diseñen, a partir de su realidad concreta, recursos disponibles y aspiraciones, procesos de mejoramiento de sus condiciones de vida. Lo anterior, guarda una estrecha relación con las propuestas del 'buen vivir', elaboradas a partir de las prácticas de la región andina de América Latina (López, 2013).

La posibilidad de intercambiar estos conocimientos con los estudiosos de la ciencia moderna se da a través del diálogo de saberes y la construcción del diálogo de saberes, implica una diversidad de creencias y valores entre los campesinos y se expresa como respeto, reconocimiento de sus experiencias de vida e interés mutuo en la comprensión de sus razones (Argueta, 2016).

El esfuerzo de crear puentes de diálogo entre diferentes sistemas de conocimiento, donde el conocimiento occidental moderno es uno más y los saberes ancestrales de pueblos y naciones que no han sido parte del desarrollo de la ciencia occidental moderna son fundamentales, hoy son reconocidos por sus aportes a la sustentabilidad alimentaria y por ser fuente para el surgimiento de nuevos paradigmas al desarrollo. En esta perspectiva se plantea el 'vivir bien' (Bolivia) o el 'buen vivir' (Ecuador) como paradigmas alternativos al desarrollo capitalista imperante en el mundo (Delgado y Rist, 2016).

Gudynas y Acosta (2011) plantean el buen vivir como una oportunidad para construir otra sociedad sustentada en la convivencia del ser humano en diversidad y armonía con la naturaleza, a partir del reconocimiento de los diversos valores culturales existentes en cada país y en el mundo. El abanico de imaginarios detrás del elusivo 'vivir bien' es bastante amplio y en general no está puesto en debate. El problema básico del 'vivir bien' es que sus difusores no han logrado vincular un programa que supuestamente surge de las cosmovisiones indígenas con las experiencias vitales de los indígenas y de las comunidades realmente existentes (Stefanoni, 2012).

Desde esta perspectiva, es necesario redoblar los esfuerzos por hacer posible la construcción de alternativas al desarrollo agrícola, desarrollo sustentable, desarrollo comunitario o también llamado etnodesarrollo (Cruz et al., 2015). La continuidad de la implementación de los saberes agrícolas en los sistemas de producción puede ser una alternativa sustentable para los campesinos.

\section{Los saberes endógenos y el desarrollo}

El pequeño productor, denominado así por la extensión de sus parcelas ( 2 ha en promedio a nivel nacional) posee el conocimiento y la habilidad para la producción agrícola (INIFAP, 2013). Por este motivo, solo hace falta el impulso para construir junto con él, un desarrollo agrícola en el sector cafetalero a partir de los saberes, recursos y perspectivas de los cafeticultores.

Sobre todo, porque debido a sus condiciones, forman un sector muy vulnerable y desfavorecido. En un estudio realizado por Cardeña (2017) en una región poblana, se encontró que los productores de café son mayormente de edad avanzada entre 41 y 60 años, lo que los limita para incorporarse a otras actividades laborales, además influye de manera negativa en la obtención de apoyos, ya sea para la producción o financiamientos. Los campesinos incorporan el café a su vida social y productiva de diversos modos, no de uno solo, aun cuando se trate de pequeños productores que comparten condiciones de producción semejantes. Y sus respuestas ante los acontecimientos también son diversas. No se puede hacer referencia a una cultura homogénea. Es importante 
ubicarnos en las dinámicas locales, las de cada poblado, dentro de la región y en la relación que los productores y sus familias establecen con el producto, con otros cultivos y actividades económicas, con los grupos involucrados en la actividad cafetalera, otros campesinos, compradores, industrializadores y funcionarios de gobierno (Ejea, 2009).

La participación de las comunidades campesinas y la utilización del reconocimiento de saberes, han resultado exitosamente en proyectos de etnodesarrollo. De acuerdo a Bonfil (1995) cualquier proyecto de etnodesarrollo consiste en una ampliación y consolidación de los ámbitos de la cultura propia; es decir, en el incremento de la capacidad de decisión del propio grupo social, tanto sobre sus recursos como sobre recursos ajenos de los que pueda apropiarse.

Este reservorio de conocimientos tradicionales, en donde se incluyen las tecnologías agrícolas, forman un sistema de producción llamado agricultura tradicional, el cual, tiene sus inicios con la domesticación de las primeras plantas cultivadas en México, hace aproximadamente nueve mil años y que fue perturbada con la conquista española en el siglo XVI. A pesar de esta intromisión, hoy en día se mantiene la agricultura tradicional como forma de producción dominante (Cruz et al., 2016).

En el caso del café, por ser un cultivo comercial, el sistema agrícola tradicional ha sido desplazado por las acciones de las nuevas políticas neoliberales, por lo que persiste un rezago en el desarrollo de las unidades de producción, debido a que estas acciones, no están adecuadas a las condiciones locales de producción, ni a las necesidades de los productores.

Ante esta situación, es importante la elaboración de una propuesta de manejo del cultivo, combinando los conocimientos o saberes agrícolas, también llamado ciencia endógena, que responde a los intereses, conocimientos y tecnologías de los productores, con la ciencia occidental, del diálogo de saberes y la transdisciplina.

\section{Conclusiones}

El estudio de los conocimientos tradicionales que iniciaron las etnociencias, ahora por medio de las ciencias endógenas está acorde a la agricultura nacional dominante en el país, permite conocer las necesidades y aspiraciones de los productores y con base en ello, proponer alternativas de solución viables para resolver los problemas del campo.

En el caso de los pequeños productores de café, existe un bagaje de conocimiento ancestral tradicional que resulta pertinente retomar y conjugar para lograr a mediano o largo plazo una agricultura sustentable con mayores beneficios a nivel de producción y calidad de vida de los productores y sus familias, que se traduce en bienestar y desarrollo a nivel de las regiones cafetaleras de México.

Esto implica el uso del diálogo de saberes entre la ciencia moderna y los saberes agrícolas, para elaborar propuestas alternativas, mejorar los sistemas de producción, lograr el bienestar de los pequeños productores y la conservación del ambiente. 
Bajo la perspectiva del buen vivir, aportada por los pueblos originarios sudamericanos, se involucra la recuperación del saber indígena y la visión de desarrollo se separa de las ideas occidentales convencionales del progreso y se apunta hacia la construcción de una idea propia de desarrollo, fundamentada en el conocimiento ancestral, basado en los conocimientos de las prácticas agrícolas llevadas a cabo en las parcelas de producción en donde han obtenido el sustento por miles de años.

Con el análisis de las investigaciones citadas es posible saber y comprender que existe un conocimiento ancestral sobre las prácticas agrícolas tradicionales que han mantenido durante años las parcelas en producción y que han abastecido de alimentos a las comunidades indígenas. Perpetuar este conocimiento y ponerlo en práctica con una metodología alternativa que incluye los saberes y avances científicos en busca de una solución sustentable.

\section{Literatura citada}

Argueta, V. A. 1997. La construcción de las etnociencias de la naturaleza y el desarrollo de los saberes biológicos de los pueblos indígenas. Tesis de maestría. Universidad Nacional Autónoma de (UNAM). México, DF. 124 p.

Argueta, V. A. 2016. El diálogo de saberes, una utopía realista. In: ciencias, diálogo de saberes y transdisciplinariedad. Aportes teórico- metodológicos para la sustentabilidad alimentaria y del desarrollo. Delgado, F. y Rist, S. (Eds.). Agruco. La Paz, Bolivia. 13-368 pp.

Arrieta, E. N. 2015. Estrategia de manejo no químico de la roya del cafeto. Centro de Investigaciones en Café. FAO. Costa Rica. 68-69 pp.

Beristain, R. B. 2001. Documento 'A'. Centro de Investigación y Desarrollo de las Regiones Cafetaleras. CRUO, Huatusco, México. 33 p.

Bonfil, B. G. 1995. Etnodesarrollo: sus premisas jurídicas, políticas y de organización. In: obras escogidas de Guillermo Bonfil Batalla. Tomo 2. Instituto Nacional de Antropología e Historia (INAH)-Instituto Nacional Indigenista (INI). 464-480 p.

CAGF. 2007. Censo Agrícola, Ganadero y Forestal.

Cardeña, B. I. 2017. Conocimiento campesino sobre la roya (Hemileia vastatrix, Berkeley \& Broome 1869) y su impacto en la producción de café en el municipio de Hueytamalco, Puebla. Tesis de maestría. Colegio de Posgraduados en Ciencias Agrícolas-Campus Puebla. $72 \mathrm{p}$.

Collado, R. J. 2016. Epistemología del sur: una visión descolonial a los objetivos de desarrollo sostenible. Sankofa. Revista da História da África e de Estudos da Diáspora Africana. 17(9):137-158.

Cruz, H. S. y Torres, C. G. 2015. El conocimiento campesino del agroecosistema cafetalero en la sierra sur de Oaxaca. Rev. Mex. Cienc. Agríc. 2:147-154.

Cruz, L. A.; Cervantes, H. J.; Ramírez, G. A.; Sánchez, G. P.; Damián, H. M. A. y Ramírez, V. B. 2015(a). La etnoagronomía en la construcción de propuestas de desarrollo rural para comunidades campesinas. Ra Ximhai. 5(11):185-194.

Cruz, L. A.; Cervantes, H. J.; Damián, H. M. A.; Ramírez, V. B. y Chávez, S. P. G. 2015(b). Etnoagronomía, tecnología agrícola tradicional y desarrollo rural. Revista de geografía agrícola. 55:75- 89. http://dx.doi.org/10.5154/r.rga.2015.55.006.

Cruz, L. A.; Cervantes, H. J. y Franco, G. A. 2016. La etnoagronomía y las necesidades de investigación, educación y vinculación con las comunidades campesinas marginadas de México. Memoria. Texcoco Estado de México. 408-409 pp. 
De Sousa, S. B. 2009. Una epistemología del sur: la reinvención del conocimiento y la emancipación social. México, siglo XXI. CLACSO. Soc. Pol. 368 p.

De Sousa, S. B. 2011. Introducción: las epistemologías del sur, Universidade de Coimbra. Foro Social Mundial. 22 p.

Delgado, F. y Rist, S. 2016. Ciencias, diálogo de saberes y transdisciplinariedad. Aportes teórico-metodológicos para la sustentabilidad alimentaria y del desarrollo Bolivia. Agruco. 41-179 pp.

Dzib, A. L. A. 1994. La tecnología de cultivo en la sierra de Zongolica, Ver. Rev. Geog. Agríc. 20:35-54.

Ejea, M. M. T. 2009. Café y cultura productiva en una región de Veracruz. Nueva Antropología. 22(70):33-56.

Escamilla, P. E. 2016. Las variedades de café en México ante el desafío de la roya. Programa mexicano del carbono, 4 breves de políticas públicas, Huatusco, Veracruz, México. Boletín Informativo. $10 \mathrm{p}$.

Escamilla, P. E. y Díaz, C. S. 2016. Sistemas de cultivo de café en México. Cenacafé, Huatusco, Veracruz. $61 \mathrm{p}$.

González, J. A. 2006. Dealing with risk: small-scale coffee production systems in Mexico. Perspectivas Latinoam. 1:1-39.

Gudynas, E. y Acosta, A. 2011. El buen vivir o la disolución de la idea del progreso, en Rojas M. (Ed). La medición del progreso y el bienestar. Propuestas desde América Latina. Foro Consultivo Científico y Tecnológico de México. 103-110 pp.

Hernández, X. E.; Inzunza, M. F.; Solano, S. B. y Brauer, G. F. 1976. Estudio de la tecnología agrícola tradicional en México. Colegio de Postgraduados en Ciencias Agrícolas, Montecillo, Estado de México. 27-30 pp.

INEGI. 2009. Instituto Nacional de Estadística, Geografía e informática. Estados Unidos Mexicanos. Censo Agropecuario 2007, VIII Censo Agrícola, Ganadero y Forestal. Aguascalientes.

INIFAP. 2013. Instituto Nacional de Investigaciones Forestales, Agrícolas y Pecuarias. Cafeticultura en la zona centro del estado de Veracruz, diagnóstico, productividad y servicios ambientales. Centro de investigación regional Golfo Centro, Teocelo, Veracruz. $36 \mathrm{p}$.

Lévi-Strauss, C. 1972. Antropología estructural. Tercera edición. Siglo XXI, de España (Ed). 187- $195 \mathrm{pp}$.

López, B. F. 2013. ¿Qué hacemos con los indios? Pueblos indígenas y desarrollo: entre las políticas gubernamentales y el 'buen vivir'. Rev. Papeles de Población. 77(19):177-192.

Moguel, P. y Toledo, V. M. 1996. El café en México, ecología, cultura indígena y sustentabilidad. Rev. Cienc. 43:40-50.

Olivé, L. 2009. Pluralismo epistemológico. Consejo Latinoamericano de Ciencias Sociales (CLACSO). La Paz, Bolivia. 302 p.

Pérez, F. Y. y Ruiz, E. F. 2017. Construyendo autogestión en la producción y conservación del café de calidad en Teocelo, Veracruz. Primera (Ed). Chapingo, Texcoco, Estado de México. $61 \mathrm{p}$.

Pérez, R. M. L. y Argueta, V. A. 2011. Saberes indígenas y diálogo intercultural. Rev. Cultura y Representaciones Sociales. 10(5):31-56.

Robles, B. H. M. 2011. Los productores de café en México: problemática y ejercicio del presupuesto. Mexican Rural Development Research Reports. Reporte. 14. 62 p. 
Stefanoni, P. 2012. ¿Y quién no querría vivir bien? Encrucijadas del cambio boliviano 9. In: crítica y emancipación. Stefanoni, P.; Alimonda, H.; Madoery, O.; Gómez, R.; Nuñez, S. O.; Herrera, F. J. y Torres, S. A. (Eds.). Núm. 7. Consejo Latinoamericano de Ciencias Sociales (CLACSO). Rev. Latinoam. Cienc. Soc. Buenos Aires, Argentina. 9-227 pp.

Vanhulst, J. y Beling, A. E. 2013. Buen vivir: la irrupción en América Latina en el campo gravitacional del desarrollo sostenible. Rev. Iberoam. Econ. Ecol. 1(21):01-14. 